\title{
Reduced dose to urethra and rectum with the use of variable needle spacing in prostate brachytherapy: a potential role for robotic technology
}

\author{
Shilpa Vyas, MD', Yi Le, PhD², Zhe Zhang, MS³, Woody Armour, PhD², Daniel Y. Song, MD²
}

\begin{abstract}
'Department of Radiation Oncology, Baylor Scott \& White Health/Texas A\&M College of Medicine, Temple, Texas, ${ }^{2}$ Department of Radiation Oncology and Molecular Radiation Sciences, The Johns Hopkins University, School of Medicine, Baltimore, Maryland, "Division of Oncology Biostatistics, The Sidney Kimmel Comprehensive, Cancer Center at Johns Hopkins, Baltimore, Maryland, USA
\end{abstract}

\begin{abstract}
Purpose: Several robotic delivery systems for prostate brachytherapy are under development or in pre-clinical testing. One of the features of robotic brachytherapy is the ability to vary spacing of needles at non-fixed intervals. This feature may play an important role in prostate brachytherapy, which is traditionally template-based with fixed needle spacing of $0.5 \mathrm{~cm}$. We sought to quantify potential reductions in the dose to urethra and rectum by utilizing variable needle spacing, as compared to fixed needle spacing.

Material and methods: Transrectal ultrasound images from 10 patients were used by 3 experienced planners to create 120 treatment plans. Each planner created 4 plan variations per patient with respect to needle positions: ${ }^{125}$ I fixed spacing, ${ }^{125} \mathrm{I}$ variable spacing, ${ }^{103} \mathrm{Pd}$ fixed spacing, and ${ }^{103} \mathrm{Pd}$ variable spacing. The primary planning objective was to achieve a prostate $V_{100}$ of $100 \%$ while minimizing dose to urethra and rectum.

Results: All plans met the objective of achieving prostate $\mathrm{V}_{100}$ of $100 \%$. Combined results for all plans show statistically significant improvements in all assessed dosimetric variables for urethra $\left(\mathrm{U}_{\max }, \mathrm{U}_{\text {mean }}, \mathrm{D}_{30}, \mathrm{D}_{5}\right)$ and rectum $\left(\mathrm{R}_{\max }\right.$ $R_{\text {mean, }}, R_{V 100}$ ) when using variable spacing. The dose reductions for mean and maximum urethra dose using variable spacing had $p$ values of 0.011 and 0.024 with ${ }^{103} \mathrm{Pd}$, and 0.007 and 0.029 with ${ }^{125} \mathrm{I}$ plans. Similarly dose reductions for mean and maximum rectal dose using variable spacing had $p$ values of 0.007 and 0.052 with ${ }^{103} \mathrm{Pd}$, and 0.012 and 0.037 with ${ }^{125}$ I plans.

Conclusions: The variable needle spacing achievable by the use of robotics in prostate brachytherapy allows for reductions in both urethral and rectal planned doses while maintaining prostate dose coverage. Such dosimetric advantages have the potential in translating to significant clinical benefits with the use of robotic brachytherapy.
\end{abstract}

Key words: prostate cancer, rectal sparing, robotic brachytherapy, urethral sparing, variable spacing.

\section{Purpose}

Although permanent prostate brachytherapy was initially described several decades ago, it has come into greater prominence over the last two decades due to various advancements, which have improved its accuracy, reproducibility, and overall efficacy. These advancements primarily include the development of transrectal ultrasound guidance for source positioning and sophisticated treatment planning software for dosimetric calculations, along with greater understanding of source loading patterns and tissue tolerance [1]. The evolution of permanent prostate brachytherapy from the previously used retropubic approach to the currently practiced transperineal, template-based, TRUS guided form has reduced morbidity, and improved implant quality and target volume coverage [2]. As confirmed by other studies, it has been determined that at least part of the urinary morbidity following brachytherapy is related to the dose received by the urethra, and current recommendations for brachytherapy treatment planning include minimizing dose to the urethra [3-6]. The correlation of rectal toxicity with doses received by anterior rectal wall is also proven by multiple previous studies $[7,8]$.

The development of robotic technology is one of the latest innovations in the ongoing process of technological advancements in the field of prostate brachytherapy. A number of medical robots are currently either under 
development or have been integrated into brachytherapy systems, with the purpose of possible optimization of source locations, more precise source placement, and resultant minimization of local tissue trauma [9-12]. Each robotic system has specific design features, but the ability to vary spacing of needles at non-fixed intervals is a common feature among all systems. Standard transperineal templates used in permanent prostate brachytherapy only allow for needles to be placed at a fixed $5 \mathrm{~mm}$ interval, limiting the possibilities for manipulation of needle placement to avoid critical structures or improve target coverage. By using the variable spacing feature achievable with robotic brachytherapy, it is feasible to maneuver the needle positions away from the organs at risk without compromising dose coverage to the target gland. In light of this emerging technology, AAPM/GEC-ESTRO Task Group 192 has reviewed the state-of-the-art in imageguided robotic brachytherapy and published guidelines regarding its use [13].

The primary intent of this study was to quantify the potential reductions in doses to urethra and rectum achievable with variable needle spacing, while maintaining dose coverage to the prostate. Our study is one of the first to ascertain the dosimetric benefits of this strategy.

\section{Material and methods}

The study was performed with approval of the Institutional Internal review board. The purpose of the study was to assess whether doses to urethra and rectum could be reduced with the use of variable needle spacing (compared to fixed needle spacing) while maintaining target volume coverage. Transrectal ultrasound images of ten patients were acquired and used to create permanent source brachytherapy plans using both standard template needle positioning as well as robotic needle positioning. Three experienced planners independently generated 4 plan variations for each patient image set, therefore creating 120 different plans in total. The 4 sets of plans created for each patient alternated between two isotopes as well as between fixed template or variable robotic spacing, i.e. a fixed-spacing ${ }^{103} \mathrm{Pd}$, fixed-spacing ${ }^{125} \mathrm{I}$, variable robotic-spacing ${ }^{103} \mathrm{Pd}$, and variable roboticspacing ${ }^{125}$ I plan. Patients were planned for the standard total dose of $145 \mathrm{~Gy}$ for ${ }^{125} \mathrm{I}$ and $125 \mathrm{~Gy}$ for ${ }^{103} \mathrm{Pd}$. For the fixed-spacing plans, a standard implantation grid with a square arrangement of $5 \mathrm{~mm}$ spaced needle holes was used, and only the set grid positions were allowed for needle positioning.

Prescription doses were 145 Gy for ${ }^{125}$ I and 125 Gy for ${ }^{103} \mathrm{Pd}$ plans, respectively. The primary planning objectives were to achieve a prostate $V_{100}$ of $100 \%$ while minimizing dose to urethra and rectum. The secondary planning objective was to minimize number of needles and number of sources. Plans utilized only intraprostatic sources, taking care to avoid needles passing through urethra. All needles were parallel to each other and orthogonal to the plane of a standard template.

Dosimetric variables that were assessed included $\mathrm{V}_{100}, \mathrm{~V}_{150}$, and $\mathrm{V}_{200}$ (prostate volume receiving $100 \%$, $150 \%$, and $200 \%$ of the prescribed dose), urethral max dose $\left(\mathrm{U}_{\text {max }}\right)$, mean dose $\left(\mathrm{U}_{\text {mean }}\right), \mathrm{D}_{30}$ and $\mathrm{D}_{5}$ (dose received by $30 \%$ and $5 \%$ of urethra), and rectal max dose $\left(R_{\max }\right)$, mean dose $\left(R_{\text {mean }}\right)$, and rectal volume receiving $100 \%$ of the prescribed dose $\left(R_{\mathrm{V} 100}\right)$. All treatment planning was performed on commercially available software (Variseed V8.0, Varian Medical Systems, Palo Alto, CA, USA). The selected source type for all cases was either TheraSeed 200 ( ${ }^{103}$ Pd; Theragenics, Norcross, GA, USA) or Best Medical model 2301 ( ${ }^{125}$ I, Best Medical Intl, Springfield, VA, USA). Source activities were $0.5 \mathrm{U}$ for ${ }^{125} \mathrm{I}$ and $2.5 \mathrm{U}$ for ${ }^{103} \mathrm{Pd}$.

\section{Statistical analysis}

Analyses were performed to compare the overall effect of robotic-spacing vs. fixed-spacing across all patients, treatment planners, and isotopes, with subset analyses to evaluate for independent effects of each of these factors upon doses to urethra and rectum. Dosimetric parameters as well as number of needles and sources were summarized using descriptive statistics and reported as mean \pm standard deviation (SD). Intraclass correlation coefficients (ICC) were used to evaluate the inter-planner reliability of dosimetric parameters. The resulting ICCs suggested moderate to substantial agreement among the planners and therefore subsequent analyses were performed on pooled data from all three planners. To account for the correlation among observations within the same subject, the method of generalized estimating equations (GEE) was applied to compare the variable of interest between the plans by assuming an exchangeable correlation structure. Patient related factors such as the volume of the prostate was adjusted for in the GEE model and interaction between isotopes used and the spacing plans was evaluated. Given the exploratory nature of this study, the $p$ values were not adjusted for multiple comparisons. All tests were two-sided and considered statistically significant at $p<0.05$. Analyses were carried out using SAS software (v. 9.2, SAS Institute, Cary, NC, USA).

\section{Results}

The prostatic volumes of these ten patients varied from $11 \mathrm{cc}$ to $53 \mathrm{cc}$, with the median being $31.7 \mathrm{cc}$. All the plans met the primary objective of achieving prostate $\mathrm{V}_{100}$ of $100 \%$. The ICCs for dosimetric parameters were in the range of $0.60-0.83$, suggesting moderate to substantial agreement among the planners. For both ${ }^{125} \mathrm{I}$ and ${ }^{103} \mathrm{Pd}$ plans, all measured variables showed a statistically significant improvement with the use of variable spacing when compared to those with the use of fixed spacing, except for $R_{\max }{ }^{103} \mathrm{Pd}$, which had only marginal statistical significance (Table 1).

The mean numbers of needles with ${ }^{103} \mathrm{Pd}$ plans were $33( \pm 9)$ and $35( \pm 8)$ for variable and fixed spacing, respectively, which were found to be significantly different despite the differences being only 1-2 needles (Table 1 ). Similarly, the mean difference in number of sources with ${ }^{125} \mathrm{I}$ plans was clinically trivial although statistically significant (Table 1). Figure 1 shows the pictorial depiction of the urethral sparing with variable-spacing plans using ${ }^{103} \mathrm{Pd}$. 
Table 1. Comparisons between fixed and variable spacing for ${ }^{103} \mathrm{Pd}$ and ${ }^{125} \mathrm{I}$ isotopes

\begin{tabular}{|c|c|c|c|c|c|c|c|c|}
\hline \multirow[t]{3}{*}{ Variable } & \multicolumn{4}{|c|}{${ }^{103} \mathrm{Pd}$} & \multicolumn{4}{|c|}{125} \\
\hline & \multicolumn{3}{|c|}{ Mean (SD) } & \multirow[t]{2}{*}{$p$ value $^{\star}$} & \multicolumn{3}{|c|}{ Mean (SD) } & \multirow[t]{2}{*}{$p$ value } \\
\hline & $\begin{array}{c}\text { Fixed } \\
\text { spacing } \\
(n=30)\end{array}$ & $\begin{array}{l}\text { Variable } \\
\text { spacing } \\
(n=30)\end{array}$ & $\%$ reduction & & $\begin{array}{c}\text { Fixed } \\
\text { spacing } \\
(n=30)\end{array}$ & $\begin{array}{l}\text { Variable } \\
\text { spacing } \\
(n=30)\end{array}$ & $\%$ reduction & \\
\hline$V_{150}(\%)$ & $72.6(5.5)$ & $69.9(5.2)$ & 3.7 & 0.028 & $56.6(7.0)$ & $53.0(6.9)$ & 6.4 & 0.004 \\
\hline$V_{200}(\%)$ & $41.3(5.5)$ & $40.2(5.0)$ & 2.7 & 0.116 & $21.5(4.3)$ & 20.4 (3.6) & 4.9 & 0.009 \\
\hline $\mathrm{U}_{\max }(\%)$ & $183.8(62.7)$ & 138 (17.5) & 24.9 & 0.024 & $151.4(29.0)$ & $136(14.0)$ & 10.2 & 0.029 \\
\hline$\cup_{\text {mean }}(\%)$ & $113.8(8.1)$ & $106.1(4.8)$ & 6.8 & 0.011 & $117.8(8.9)$ & $113.8(8.8)$ & 3.4 & 0.007 \\
\hline $\mathrm{UD}_{30}(\%)$ & $123(10.5)$ & $115.5(6.8)$ & 6.1 & 0.016 & $126.3(5.9)$ & $121.7(5.3)$ & 3.6 & 0.004 \\
\hline $\mathrm{UD}_{5}(\%)$ & $140.2(16.2)$ & $124.8(9.1)$ & 11.0 & 0.008 & $134.3(7.6)$ & $127.4(7.0)$ & 5.1 & 0.002 \\
\hline$R_{\max }(\%)$ & $106(32.1)$ & $95.8(19.8)$ & 9.6 & 0.052 & 107.4 (22.9) & $98.5(15.5)$ & 8.3 & 0.037 \\
\hline$R_{\text {mean }}(\%)$ & $46.2(9.7)$ & $44.1(9.3)$ & 4.5 & 0.007 & $57.8(8.4)$ & $56.5(8.6)$ & 2.2 & 0.012 \\
\hline $\mathrm{R}_{\mathrm{V} 100}(\%)$ & $0.03(0.05)$ & $0.02(0.03)$ & 33.3 & 0.039 & $0.03(0.04)$ & $0.01(0.02)$ & 66.7 & 0.029 \\
\hline \# Needles & $35(8)$ & $33(9)$ & 5.7 & 0.012 & $32(6)$ & $31(7)$ & 3.1 & 0.129 \\
\hline \# Sources & $75(22)$ & $74(22)$ & 1.3 & 0.086 & $64(16)$ & $63(16)$ & 1.6 & 0.022 \\
\hline
\end{tabular}

$V_{150}, V_{200}$ - percentage of prostate volume receiving $150 \%$ and $200 \%$ of prescribed dose, respectively; $U_{\text {max }}, U_{\text {mean }}-$ maximum and mean percentage of prescription dose received by urethra; $U D_{30}, U D_{5}$ - minimum percent dose received by $30 \%$ and $5 \%$ of urethra respectively; $R_{\text {max }}, R_{\text {mean }}-$ maximum and mean percentage of prescription dose received by rectum; $R_{V 100}$ - percentage of rectal volume receiving $100 \%$ of prescription dose

*Obtained using the generalized estimating equations (GEE) modeling approach
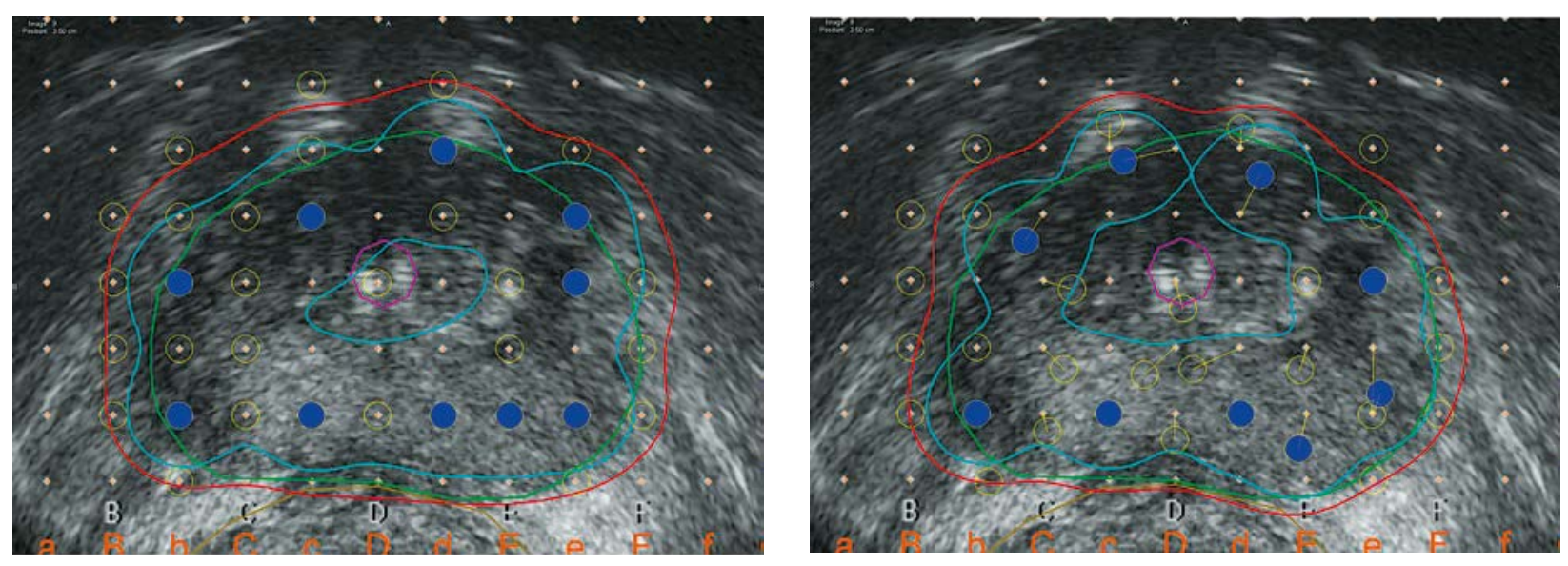

Fig. 1. Depiction of dose distribution in fixed needle spacing and variable needle spacing plans. Transverse section of ultrasound image at mid-prostate level planned with ${ }^{103} \mathrm{Pd}$ seeds using fixed needle spacing plan (left) and variable needle spacing plan (right). The urethral sparing is well visualized with the variable spacing plan by assessing the distance between the urethra and the $150 \%$ isodose line.

Key: prostate gland - green; urethra - magenta; $100 \%$ isodose line - red; $150 \%$ isodose line - turquoise; needle/source positions (sources within currently visualized axial plane) - blue; other needle positions (sources not in currently visualized axial plane) - empty circles.

\section{Discussion}

In the current study we were able to demonstrate that urethra doses would be reduced with variable needle spacing. Although statistically significant dose reductions were seen for both isotopes and for all dosimetric parameters assessed, greater reductions were seen for urethra max doses (as compared to $\mathrm{D}_{5}$ and $\mathrm{D}_{30}$ ), and percent reductions were larger for ${ }^{103} \mathrm{Pd}$ compared to ${ }^{125} \mathrm{I}$ for all parameters. Although rectal doses were also statistically significantly lower for variable spacing plans, the absolute rectal doses were low and the magnitude of differences relatively small. Due to the variability of actual clinical dosimetric outcomes vs. pre-treatment plans, it remains to be seen whether the dosimetric advantages observed 
in this study will translate into dose reductions in clinical practice.

Several analyses have supported the role of urethral dose in post-brachytherapy urinary morbidity, some of which formed the basis for prescribing dose limitations for maximum urethral dose to prevent severe urinary symptoms [3,4]. The early experience of Wallner et al. [14] demonstrated an intimate relationship between urethral dose and urinary morbidity. Patients with RTOG Grades 0-1 or Grades 2-3 urinary morbidity had average maximum urethral doses of 447 Gy ( $279 \%$ minimum peripheral dose [mPD]) and $592 \mathrm{~Gy}(370 \% \mathrm{mPD})$, respectively [14]. Subsequently, some studies have found lack of correlation between urethral doses and acute urinary symptoms, especially acute urinary retention and stated that the pre-implant parameters were more important [15-17]. It is known that acute urinary retention, which has been most strongly correlated with prostate volume and pre-existing baseline symptoms of urinary retention, is only one component of the entire spectrum of urinary morbidity. In a recently published study by Register et al. [18], MRI was used in delineating the external and internal urethral sphincters (EUS \& IUS) and the doses received by both were calculated in patients undergoing monotherapy with ${ }^{125} \mathrm{I}$ for intermediate-risk localized prostate cancer. They showed that urinary function declined when IUS V285 was $0.4 \%$. Other urinary symptoms of irritation, bother, and urgency worsened as well with increasing doses to IUS and EUS. The authors concluded that the doses to the IUS and EUS on MRI/CT predicted worse urinary function, with greater bother, irritative symptoms, and urgency [18]. Pinkawa et al. [19] analyzed health-related quality of life after prostate brachytherapy and recommended a prostate $\mathrm{D}_{90}<170 \mathrm{~Gy}$ and a base of seminal vesicle $\mathrm{D}_{10}<190 \mathrm{~Gy}$ (as an indicator of the dose to the bladder neck and urethral sphincter) to maintain a satisfactory urinary function. They found that doses higher than the threshold of $\mathrm{D}_{10}$ led to higher rates of pad usage, worse urinary bother score and urinary function score during the acute period [19]. In another study by Allen et al. [15], regional urethral dosimetry was analyzed to predict urinary morbidity. Their results demonstrate that linearly segmented urethral doses do not predict for IPSS resolution but in multivariate analyses for ${ }^{125} \mathrm{I}$ isotope the maximum dose received by the prostate apex and genitourinary diaphragm predicted the greatest increase in IPSS [15]. For the entire cohort, the maximum IPSS was best predicted by preimplant IPSS and the maximum apical urethral dose. Conversely, Hathout et al. correlated dose to bladder neck with urinary toxicity following brachytherapy [20]. Although there remains uncertainty with regard to the specific region of the urethra most correlated with urinary toxicity, the need for dose-sparing of urethra is a well-accepted concept and embedded in consensus guidelines for prostate brachytherapy $[5,6]$.

In our study, the use of variable spacing in needle positioning helped reduce the urethral dose parameters significantly. We elected to utilize the most commonly utilized urethral dosimetric parameters of interest. With different planners using their own individual planning techniques, we were able to create significant differenc- es in the urethral $\mathrm{D}_{30}$ and $\mathrm{D}_{5} \%$ doses when compared with the fixed spacing plans. The difference in doses was greater with the use of ${ }^{103} \mathrm{Pd}$ seeds, which may be expected because of the lower average energy of photons from ${ }^{103} \mathrm{Pd}$ causing a more rapid falloff of dose, leading to greater impact due to seed position changes as compared to ${ }^{125}$ I $[21,22]$.

The rectal doses in our study were low in general and respected published rectal dose constraints. Our mean rectal $\mathrm{V}_{100}$ for both fixed spacing and variable spacing plans was much less than the limit prescribed for rectal toxicity by previous studies $[7,8,23]$. The fact that most of the studies have correlated increasing doses received by the rectal wall to the advent of rectal complications leaves room for tactics to further reduce rectal doses. Most of the rectal dose parameters were significantly less with the use of variable spacing in both ${ }^{125} \mathrm{I}$ and ${ }^{103} \mathrm{Pd}$ seeds, and this difference was consistently maintained with all planners. Hence, it would be safe to assume that using the variable spacing feature of robotic brachytherapy, rectal dose reduction to a significant extent is possible, irrespective of the isotope used.

The number of needles used in the implant procedure have been implicated in increasing the urinary symptoms proportionately, the rationale being the possible trauma-related morbidity due to needle puncture, especially in the bladder neck and bladder trigone area [24-27]. Steggerda et al. [28] were able to substantially reduce the number of needles by manipulating the inter-seed spacing and by increasing the seed strength. They do, however, acknowledge the possibilities for increasing the $V_{200}$ of prostate and also for unpredictably high dose to organs at risk using this strategy. Although reduction of number of needles was a secondary goal in our study, we observed a small but statistically significant difference for ${ }^{103} \mathrm{Pd}$ plans. Such difference being only 2 needles, however, is not expected to be clinically relevant in the present scenario. Subjectively, we observed that if one used variable spacing without attempting to lower doses to urethra and rectum, fewer needles could be used, corroborating the experience of Steggerda et al. However, when attempting to reduce doses to critical structures (the primary goal of our study), it was difficult to reduce the number of needles as well. Although it remains to be determined whether dose to urethra or number of needles has greater effect on urinary morbidity, our results combined with those of Steggerda et al. [28] suggest that variable spacing could be utilized toward achieving either goal.

Similarly, prior studies have implicated the number of sources used as one of the causes for acute urinary retention $[27,29]$. We did achieve a small difference in the number of sources used, which though reaching statistical significance for ${ }^{125} \mathrm{I}$, would not appear to be clinically relevant.

The current decade is seeing the development of various robotic systems and automation tools in medicine. Several groups have described their robotic systems developed for utilization in prostate brachytherapy, although most have not been tested clinically [9-11]. Several of these systems can accommodate various needle drivers for different percutaneous interventions such as biopsy, thermal ablation, or brachytherapy. Some have novel 
features such as compatibility with an MRI environment [10-12,30,31]. Similarly, robots are also being developed for brachytherapy in other sites such as lung $[32,33]$.

In a prior work, we described our pilot clinical testing of a robotic needle guide [12]. This study demonstrated the clinical feasibility of a robotic needle positioner for use in permanent prostate brachytherapy. Optical tracking showed minor sub-millimeter errors (mean error, $0.49 \mathrm{~mm}$; standard deviation, $0.33 \mathrm{~mm}$ ) in robotic needle guide movement. Fine adjustments in needle positioning were found to be possible when tissue deflection of needles was encountered; adjustments were performed in 54/179 (30.2\%) needles placed, with $36 / 179$ (20.1\%) adjustments being above $2 \mathrm{~mm}$. Twenty-seven insertions were intentionally altered to positions between the standard template grid to improve the dosimetric plan or avoid critical structures. Our experience suggested clinical utility for such robots in performing less than $5 \mathrm{~mm}$ movements to correct for tissue deflection, as well as for minor adjustment of seed positions based on real-time dosimetry feedback in order to reduce dose to organs at risk. Another feature realized during our initial robotic brachytherapy experience was the inherent ease of manipulation of seed and needle positions in positions not confined to the standard $5 \mathrm{~mm}$ template spacing. Although other techniques to vary needle positions have been described, such as bent needles or the use of either a finger or a dedicated needle adjustment tool (aka 'Diddler') placed between template and perineum, such methods utilize non-quantitative effects on the needle with variable results on the needle trajectory [34].

Other noteworthy works have evaluated the potential use of angled needles, another capability which is offered by robotic brachytherapy. Specifically, Fu et al. evaluated the possibility of using a hybrid model of both angled and standard needle positioning in order to overcome the challenge of pubic arch interference [35]. In other work, the same group evaluated the potential for alternative implant patterns such as conical or biconical needle arrangements using robotic brachytherapy, showing that with inverse optimization, such approaches were feasible [36,37].

It remains to be seen whether the dosimetric differences portrayed in our work would be realized in a clinical setting, where variables such as tissue deflection of needles, intraoperative and postoperative edema, and variations between planned vs. actual source placements would cause changes from the original dosimetric plan. However, it is reasonable to expect that the ability to start with a dosimetrically superior plan would result in the execution of a treatment which is also dosimetrically superior in the end. Furthermore, the advantages of greater adaptability of needle position in response to alterations in intraoperative vs. planned source positions, as described in our initial clinical experience, may add to the benefits described herein.

\section{Conclusions}

The use of variable spacing allows for reductions in both urethral and rectal doses, while maintaining adequate prostate dose coverage. These results suggest that variable needle positioning, one of the inherent advantages of utilizing robotic technology for brachytherapy, may have dosimetric advantages and consequently, potential clinical benefits in terms of reduced treatment toxicity.

\section{Disclosure}

Authors report no conflict of interest.

\section{References}

1. Acher PL, Morris SL, Popert RJ et al. Permanent prostate brachytherapy: a century of technical evolution. Prostate Cancer Prostatic Dis 2006; 9: 215-220.

2. Zelefsky MJ, Yamada Y, Marion C et al. Improved conformality and decreased toxicity with intraoperative computer-optimized transperineal ultrasound-guided prostate brachytherapy. Int J Radiat Oncol Biol Phys 2003; 55: 956-963.

3. Merrick GS, Butler WM, Tollenaar BG et al. The dosimetry of prostate brachytherapy-induced urethral strictures. Int J Radiat Oncol Biol Phys 2002; 52: 461-468.

4. Thomas C, Keyes M, Liu M et al. Segmental urethral dosimetry and urinary toxicity in patients with no urinary symptoms before permanent prostate brachytherapy. Int J Radiat Oncol Biol Phys 2008; 72: 447-455.

5. Nath R, Bice WS, Butler WM et al. American Association of Physicists in Medicine. AAPM recommendations on dose prescription and reporting methods for permanent interstitial brachytherapy for prostate cancer: report of Task Group 137. Med Phys 2009; 36: 5310-5322.

6. Davis BJ, Horwitz EM, Lee WR et al. American Brachytherapy Society consensus guidelines for transrectal ultrasoundguided permanent prostate brachytherapy. Brachytherapy 2012; 11: 6-19.

7. Snyder KM, Stock RG, Hone SM et al. Defining the risk of developing grade 2 proctitis following 125I prostate brachytherapy using a rectal dose-volume histogram analysis. Int J Radiat Oncol Biol Phys 2001; 50: 335-341.

8. Waterman FM, Dicker AP. Probability of late rectal morbidity in 125I prostate brachytherapy. Int J Radiat Oncol Biol Phys 2003; 55: 342-353.

9. Yu Y, Podder TK, Zhang YD et al. Robotic system for prostate brachytherapy. Comput Aided Surg 2007; 12: 366-370.

10. Stoianovici D, Song D, Petrisor D et al. "MRI Stealth" robot for prostate interventions. Minim Invasive Ther Allied Technol 2007; 16: 241-248.

11. Muntener M, Patriciu A, Petrisor D et al. Transperineal prostate intervention: robot for fully automated MR imaging system description and proof of principle in a canine model. Radiology 2008; 247: 543-549.

12. Song DY, Burdette EC, Fiene J et al. Robotic needle guide for prostate brachytherapy: clinical testing of feasibility and performance. Brachytherapy 2011; 10: 57-63.

13. Podder TK, Beaulieu L, Caldwell B et al. American Association of Physicists in Medicine Brachytherapy Subcommittee and Therapy Physics Committee; Groupe Européen de Curiethérapie-European Society for Radiotherapy \& Oncology BRAPHYQS Subcommittee. AAPM and GEC-ESTRO guidelines for image-guided robotic brachytherapy: report of Task Group 192. Med Phys 2014; 41: 101501.

14. Wallner K, Roy J, Harrison L. Dosimetry guidelines to minimize urethral and rectal morbidity following transperineal I-125 prostate brachytherapy. Int J Radiat Oncol Biol Phys 1995; 32: 465-471.

15. Allen ZA, Merrick GS, Butler WM et al. Detailed urethral dosimetry in the evaluation of prostate brachytherapy-relat- 
ed urinary morbidity. Int J Radiat Oncol Biol Phys 2005; 62: 981-987.

16. Neill M, Studer G, Le L et al. The nature and extent of urinary morbidity in relation to prostate brachytherapy urethral dosimetry. Brachytherapy 2007; 6: 173-179.

17. Crook J, McLean M, Catton C et al. Factors influencing risk of acute urinary retention after TRUS-guided permanent prostate seed implantation. Int J Radiat Oncol Biol Phys 2002; 52: 453-460.

18. Register SP, Kudchadker RJ, Levy LB et al. An MRI-based dose-response analysis of urinary sphincter dose and urinary morbidity after brachytherapy for prostate cancer in a phase II prospective trial. Brachytherapy 2013; 12: 210-216.

19. Pinkawa M, Fischedick K, Piroth MD et al. Health-related quality of life after permanent interstitial brachytherapy for prostate cancer: correlation with postimplant CT scan parameters. Strahlenther Onkol 2006; 182: 660-665.

20. Hathout L, Folkert MR, Kollmeier MA et al. Dose to the bladder neck is the most important predictor for acute and late toxicity after low-dose-rate prostate brachytherapy: implications for establishing new dose constraints for treatment planning. Int J Radiat Oncol Biol Phys 2014; 90: 312-319.

21. Nath R, Meigooni AS, Melillo A. Some treatment planning considerations for 103Pd and 125I permanent interstitial implants. Int J Radiat Oncol Biol Phys 1992; 22: 1131-1138.

22. Meigooni AS, Nath R. A comparison of radial dose functions for $103 \mathrm{Pd}, 125 \mathrm{I}, 145 \mathrm{Sm}, 241 \mathrm{Am}, 169 \mathrm{Yb}$, 192Ir, and 137Cs brachytherapy sources. Int J Radiat Oncol Biol Phys 1992; 22: 125-130.

23. Albert M, Song JS, Schultz D et al. Defining the rectal dose constraint for permanent radioactive seed implantation of the prostate. Urol Oncol 2008; 26: 147-152.

24. Eapen L, Kayser C, Deshaies Y et al. Correlating the degree of needle trauma during prostate brachytherapy and the development of acute urinary toxicity. Int J Radiat Oncol Biol Phys 2004; 59: 1392-1394

25. Vargas C, Ghilezan M, Hollander M et al. A new model using number of needles and androgen deprivation to predict chronic urinary toxicity for high or low dose rate prostate brachytherapy. J Urol 2005; 174: 882-887.

26. Keyes M, Schellenberg D, Moravan V et al. Decline in urinary retention incidence in 805 patients after prostate brachytherapy: the effect of learning curve? Int J Radiat Oncol Biol Phys 2006; 64: 825-834.

27. Keyes M, Miller S, Moravan V et al. Predictive factors for acute and late urinary toxicity after permanent prostate brachytherapy: long-term outcome in 712 consecutive patients. Int J Radiat Oncol Biol Phys 2009; 73: 1023-1032.

28. Steggerda MJ, van der Poel HG, Moonen LM. Minimizing the number of implantation needles for prostate (125)I brachytherapy: an investigation of possibilities and implications. Brachytherapy 2010; 9: 319-327.

29. Lee N, Wuu CS, Brody R et al. Factors predicting for post-implantation urinary retention after permanent prostate brachytherapy. Int J Radiat Oncol Biol Phys 2000; 48: 1457-1460.

30. Muntener M, Patriciu A, Petrisor D et al. Magnetic resonance imaging compatible robotic system for fully automated brachytherapy seed placement. Urology 2006; 68: 1313-1317.

31. Song SE, Cho NB, FIscher G et al. Development of a Pneumatic Robot for MRI-guided Transperineal Prostate Biopsy and Brachytherapy: New Approaches. IEEE Int Conf Robot Autom 2010; 2010: 2580-2585.

32. Trejos AL, Lin AW, Pytel MP et al. Robot-assisted minimally invasive lung brachytherapy. Int J Med Robot 2007; 3: 41-51.

33. Lin AW, Trejos AL, Mohan S et al. Electromagnetic navigation improves minimally invasive robot-assisted lung brachytherapy. Comput Aided Surg 2008; 13: 114-123.
34. Wallner K, Blasko J, Dattoli MJ. Prostate brachytherapy made complicated. $2^{\text {nd }}$ edition. SmartMedicine Press, 2001.

35. Fu L, Ng WS, Liu H et al. Bouquet brachytherapy: feasibility and optimization of conically spaced implants. Brachytherapy 2005; 4: 59-63.

36. Fu L, Liu H, Ng WS et al. Hybrid dosimetry: feasibility of mixing angulated and parallel needles in planning prostate brachytherapy. Med Phys 2006; 33: 1192-1198.

37. Cunha JA, Hsu IC, Pouliot J. Dosimetric equivalence of nonstandard HDR brachytherapy catheter patterns. Med Phys 2009; 36: 233-239. 